
Military Technical College, Kobry El-Kobbah, Cairo, Egypt

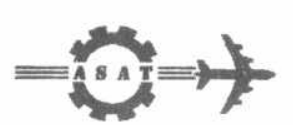

$9^{\text {th }}$ International Conference

On Aerospace Sciences \&

Aviation Technology

\title{
MAXIMIZING THE ANGULAR TRACKING ERROR INDUCED BY PHASE-FRONT DISTORTION JAMMING
}

\author{
ALADIN ASSISI.
}

\begin{abstract}
In this paper we study different factors affecting the angular tracking error of a modern radar homing missile induced by phase front distortion jamming. The normalized induced angular error due to jamming is defined as an objective function to be maximized. An analytical formula for its computation is adopted and the effect of each factor on that function is studied analytically and numerically. Those factors related to the self-protection jamming system parameters are studied as an approach to optimize the system specification, whereas the other factors, which are out of control, are studied to minimize their negative effects on the jamming performance. It is not enough just to maximize the induced angular error to get a successful deception effect on a homing-guided missile. The induced error has to be timevariant and to exist for the longest possible periods of time. That is why we tried also to maximize the ranges of variation of different parameters that guarantee a certain minimum desired value for the induced error. The results can be considered as a basis for the jamming system specification and design.
\end{abstract}

\section{KEY WORDS}

Phase front distortion, induced angular error, intended and effective phase differences, amplitude ratio, lateral distance, missile-target range, viewing angle.

\section{NOMENCLATURE}

Effective phase difference between the two jamming signals at the missile receiver input $\left(\varphi_{\text {eff }}\right)$, intended phase difference $\varphi_{12}$, additional phase shift due to path difference between the two waves $\psi$, source resolution angle $\alpha_{\text {f }}$, amplitude ratio $\gamma$,

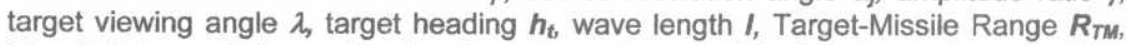
lateral distance between the two jamming antennas $d$, Induced angular error $\left[\beta_{J}=\right.$ $\left.\lambda_{\text {measured }}-\lambda_{\text {real }}\right]$, normalized angular error $\left(\beta_{j} / \alpha_{j}=\xi\right)$. and $\Delta \varphi_{\max }$ is the maximum possible phase variation range to guarantee a given value of $\xi_{m}$.

- PhD., Egyptian Armed Forces 
that the normalized angular error $\left(\beta_{j} / \alpha_{j}=\xi\right)$ neither varies with $d$ nor with the range $\boldsymbol{R}_{T M}$. For sinall values of $\beta_{j}$, we can approximate and re-arrange (1) to get:

$$
2 \xi=\left(\frac{2 \beta_{j}}{\alpha_{j}}\right) \cong\left(\frac{1-\gamma^{2}}{\gamma^{2}+2 \gamma \cos \varphi_{e f f}+1}\right)
$$

This approximation can be adopted in most cases of the angular deception problem; since the maximum attainable values of the induced error cannot exceed half the beam width [1]. Typical values of $\beta_{j} \leq 6^{\circ}$ are applicable on most homing missile systems and still practically effective. Fig. 3 shows the variation of $\xi$ with $\varphi_{\text {eff }}$ at different values of $\gamma$. As $\gamma$ exceeds unity the normalized error becomes negative. This means that the angle tracking system goes towards the jamming source with higher amplitude, which is a logical consequence.

2. From equation (6) we can easily deduce that the maximum error occurs when the effective phase difference arrives at $180^{\circ}$. If we substitute $\varphi_{\text {eff }}=180^{\circ}$ in (6) we get

$$
2 \xi_{\max }=\left(\frac{2 \beta_{j}}{\alpha_{j}}\right)_{\max } \cong\left(\frac{1-\gamma^{2}}{\gamma^{2}-2 \gamma+1}\right)=\frac{1+\gamma}{1-\gamma}
$$

Fig. 4 shows the relation between the maximum value of the normalized induced error $\xi_{\max }$ and the amplitude ratio $\gamma$. It is evident that for nearly equal amplitudes the function approaches an asymptote and that goes negative values of $\xi$ for $\gamma>1$.

3. The normalized angular error magnitude $/ \xi /$ increases as $\gamma$ approaches unity. At the same time the permissible range of phase variation around $180^{\circ}$ to guarantee a certain angular error changes. Assume that the minimum required value for the normalized error $\left(\beta_{j} / \alpha_{j}\right)$ is $\xi_{m}$. Substituting in (6) and rearranging we get:

$$
\cos \left(\varphi_{\text {eff }}\right)=\frac{\gamma^{2} \cdot\left(2 \xi_{m}+1\right)+\left(2 \xi_{m}-1\right)}{-4 \gamma \cdot \xi_{m}}
$$

Solving the quadratic equation in $\gamma$ we can get the condition to guarantee $\xi \geq \xi_{m}$ :

$$
\frac{2 \xi_{m}-1}{2 \xi_{m}+1} \leq \gamma \leq 1
$$

For a given value of $\xi_{m}$ we can plot $\cos \left(\varphi_{\text {eff }}\right)$ (and consequently $\varphi_{\text {eff }}$ ) vs. $\gamma$ and get the corresponding range of values for $\varphi_{\text {eff. }}$ This is shown in Fig.5. For example, in order that $\xi$ exceeds 20, $\gamma$ must exceed 0.9512 . For $\gamma=0.96$ the permissible range of phase variation to guarantee $\xi \geq \mathbf{2 0}$ is $180 \pm 1.2^{\circ}$. Note that this range of $\varphi_{\text {eff }}$ values around $180^{\circ}$ is doubled since the function arccos is double-valued. It is clear from 


$$
\varphi_{\text {eff }}=\psi+\varphi_{12}=\left(\frac{2 \pi d \sin \left(\lambda+h_{t}\right)}{l}\right)+\varphi_{12}
$$

$\psi$ is the additional phase shift due to path difference between the two waves $\left(\varphi_{12}\right)$ is the intended phase shift between the two coherent sources $I$ is the wave length [in meters].

\section{STUDYING THE EFFECTS OF DIFFERENT FACTORS}

To study the effect of each factor on the jamming-induced error $\beta_{j}$ we have to isolate the other factors or assign them fixed values. We shall start with the jamming system parameters; since their optimization means a correct specification of that system. Other parameters, such as the viewing angle $\lambda$, the wavelength $I$ and the Range $R_{T M}$ will also be studied to minimize their negative effects on the deception process.

\section{Studying the Effect of the Jamming System Parameters}

The main jamming system parameters affecting the induced angular error are the intended phase difference $\left(\varphi_{12}\right)$ between the two sources, their amplitude ratio $\gamma$ and lateral distance $\boldsymbol{d}$. To study the effects of these parameters we proceed as follows:

- Assign zero values to $\lambda$ and $\boldsymbol{h}_{t}$. This is a practical assumption in air-to-air missile attacks. It isolates all sources of error other than those due to jamming. Under this assumption $\varphi_{\text {eff }}$ equals the intended phase difference $\left(\varphi_{12}\right)$.

- Vary the ratio between the lateral distance $d$ and the radar wavelength $I$ and study the effect of this variation on the resulting error $\beta_{i}$. Typical values are assigned to the range $\boldsymbol{R}_{T M}$. Those values will also be varied to study their effect.

Substituting zero values for $\lambda$ and $\boldsymbol{h}_{\boldsymbol{t}}$ in the formulae (1) and (2) we get:

$$
\begin{aligned}
\tan \left(\beta_{j}\right) & =\left(\frac{\alpha_{j}}{2}\right) \cdot\left(\frac{1-\gamma^{2}}{\gamma^{2}+2 \gamma \cos \varphi_{12}+1}\right) \\
\alpha_{j} & =d / R_{T M}
\end{aligned}
$$

\section{Results of the Study}

1. It is evident from (4) and (5) that the induced error is proportional to the ratio between the lateral distance $\boldsymbol{d}$ and the range $\boldsymbol{R}_{T M}$. Fig. 2 . shows the variation of $\beta_{j}$ with $\varphi_{\text {eff }}$ and $\gamma$ at two different values of $\boldsymbol{d}$ when $\boldsymbol{R}_{T M}$ is 10 [km]. If we normalize the induced error $\beta_{j}$ to the source resolution angle $\alpha_{j}$ we get the important conclusion 
that the normalized angular error $\left(\beta_{L} / \alpha_{j} \equiv \xi\right)$ neither varies with $d$ nor with the range $\boldsymbol{R}_{T M}$. For small values of $\beta_{j}$, we can approximate and re-arrange (1) to get:

$$
2 \xi=\left(\frac{2 \beta_{j}}{\alpha_{j}}\right) \cong\left(\frac{1-\gamma^{2}}{\gamma^{2}+2 \gamma \cos \varphi_{e j}+1}\right)
$$

This approximation can be adopted in most cases of the angular deception problem; since the maximum attainable values of the induced error cannot exceed half the beam width [1]. Typical values of $\beta_{j} \leq 6^{\circ}$ are applicable on most homing missile systems and still practically effective. Fig. 3 shows the variation of $\xi$ with $\varphi_{\text {eff }}$ at different values of $\gamma$. As $\gamma$ exceeds unity the normalized error becomes negative. This means that the angle tracking system goes towards the jamming source with higher amplitude, which is a logical consequence.

2. From equation (6) we can easily deduce that the maximum error occurs when the effective phase difference arrives at $180^{\circ}$. If we substitute $\varphi_{\text {eff }}=180^{\circ}$ in (6) we get

$$
2 \xi_{\max }=\left(\frac{2 \beta_{j}}{\alpha_{j}}\right)_{\max } \cong\left(\frac{1-\gamma^{2}}{\gamma^{2}-2 \gamma+1}\right)=\frac{1+\gamma}{1-\gamma}
$$

Fig. 4 shows the relation between the maximum value of the normalized induced error $\xi_{\max }$ and the amplitude ratio $\gamma$. It is evident that for nearly equal amplitudes the function approaches an asymptote and that goes negative values of $\xi$ for $\gamma>1$.

3. The normalized angular error magnitude $/ \xi /$ increases as $\gamma$ approaches unity. At the same time the permissible range of phase variation around $180^{\circ}$ to guarantee a certain angular error changes. Assume that the minimum required value for the normalized error $\left(\beta_{j} / \alpha_{j}\right)$ is $\xi_{m}$. Substituting in (6) and rearranging we get:

$$
\cos \left(\varphi_{\text {eff }}\right)=\frac{\gamma^{2} \cdot\left(2 \xi_{m}+1\right)+\left(2 \xi_{m}-1\right)}{-4 \gamma \cdot \xi_{m}}
$$

Solving the quadratic equation in $\gamma$ we can get the condition to guarantee $\xi \geq \xi_{m}$ :

$$
\frac{2 \xi_{m}-1}{2 \xi_{m}+1} \leq \gamma \leq 1
$$

For a given value of $\xi_{m}$ we can plot $\cos \left(\varphi_{\text {eff }}\right.$ ) (and consequently $\varphi_{\text {eff }}$ ) vs. $\gamma$ and get the corresponding range of values for $\varphi_{\text {eff. }}$ This is shown in Fig.5. For example, in order that $\xi$ exceeds $20, \gamma$ must exceed 0.9512 . For $\gamma=0.96$ the permissible range of phase variation to guarantee $\xi \geq 20$ is $180 \pm 1.2^{\circ}$. Note that this range of $\varphi_{\text {eff }}$ values around $180^{\circ}$ is doubled since the function arccos is double-valued. It is clear from 
Fig. 5 that at a certain value of $\gamma$ the permissible range of phase variation becomes a maximum. We can obtain his value by differentiating (8) and equating with zero. The result is:

$$
\gamma_{m}=\sqrt{\frac{2 \xi-1}{2 \xi+1}}
$$

Let us define the maximum possible phase variation range to guarantee a given value of $\xi_{m}$ and call it $\Delta \varphi_{\max }=2\left(\varphi_{m}-1809\right.$. Fig.6 shows the corresponding values of $\gamma$ at which those maximum phase variations are permissible. It is evident from the figure that the higher the amplitude ratio $\gamma$ the higher the possible relative error $\xi$ and the smaller the allowance $\Delta \varphi_{\max }$.

\section{Studying the Effect of the Viewing Angle}

To study this effect we assign a value $180^{\circ}$ to the intended phase shift and assign $I$, $\boldsymbol{d}$ and $\boldsymbol{R}_{T M}$ typical practical values. We still assume zero target heading and take the amplitude ratio $\gamma$ as a parameter. Substituting in (3) and (6) we get:

$$
2 \xi=\left(\frac{2 \beta_{j}}{\alpha_{j}}\right) \cong \frac{1-\gamma^{2}}{\gamma^{2}+2 \gamma \cos \left(\frac{2 \pi d \sin \lambda}{l}\right)+1}
$$

We can plot $\boldsymbol{\xi}$ vs. $\lambda$ as shown in Fig.7a. It is evident that the relation is independent of $\boldsymbol{R}_{T M}$. If we normalize $\lambda$ to $\alpha_{j}$ we get the families of curves shown in Fig.7b. for two different values of $\boldsymbol{R}_{T M}$. It is evident from Fig.7 that the effect of the parameter $\gamma$ is the same as discussed above. We can easily recognize from (11) that $\xi$ is a periodic function of $\lambda$. It has a maximum for $\lambda=\sin ^{-1}\{(2 n+1) 1 / 2 d\}, n=0,1,2, \ldots \ldots$ when $\cos \Psi$ equals -1 . It gets its minimum values for $\lambda=\sin ^{-1}(n I / d), n=0,1,2, \ldots \ldots$. when $\cos \Psi$ equals 1 . Substituting with these values of $\lambda$ we get the variation range of $\xi$.

$$
\frac{1-\gamma}{1+\gamma} \leq 2 \xi \leq \frac{1+\gamma}{1-\gamma}
$$

The periodic behavior of $\xi$ vs. $\lambda$ is shown in Fig. 8 for $R_{7 M}=20[\mathrm{~km}], \mathbf{d} / \mathbf{l}=40$ and 80 , $\gamma=0.4$ to $0.8, \varphi_{12}=180^{\circ}$ and $0^{\circ}$. The antenna beamwidth is assumed $10^{\circ}$ which is a practical value. Note that the variation of $\beta_{j}$ with $\lambda$ is modulated with the variation of $\alpha_{j}$, while that of $\xi$ has constant amplitude. Note also that the $\varphi_{\text {eff }}=\varphi_{12}$ at zero viewing
angle. 


\section{SUMMARY OF RESULTS}

The normalized induced angular error $\xi$ is independent of $\boldsymbol{R}_{T M}$. It has a maximum at $\varphi_{\text {eff }}=180^{\circ}$. This maximum value increases with the amplitude ratio $\gamma$, goes to an asymptote when $\gamma$ approaches unity and reverses polarity for $\gamma>1$. The variation of $\xi$ around its maximum is symmetrical with the variation of $\varphi_{\text {eff }}$ around $180^{\circ}$. The rate of such a variation depends mainly on $\gamma$. For each required value of $\xi$ there is a certain value of $\gamma$ for maximum allowance of $\varphi_{\text {eff }}$ around $180^{\circ}$. This critical value increases with $\xi$ but the allowed range around $180^{\circ}$ (to be called $\Delta \varphi_{\max }$ ) decreases correspondingly. The lower the ratio $\gamma$ the wider that allowance $\Delta \varphi_{\max }$ becomes. Since $\varphi_{\text {eff }}$ is a periodic function of the viewing angle $\lambda, \xi$ is also periodic in $\lambda$. The period increases with the ratio $I / d$. The corresponding allowed range of viewing angle variation to guarantee a certain required error $\Delta \lambda_{\max }$ also increases with that ratio, while the maximum error value depends only on the amplitude ratio $\gamma$.

\section{CONCLUSION}

A detailed study has been done on different factors affecting the induced error in a missile angle tracking system by phase-front distortion jamming. The jamming system parameters $\varphi_{12}, \gamma$ and $d / l$ affect the maximum error, the phase allowance $\Delta \varphi_{\max }$ and the error variation period with respect to the viewing angle. But there are other factors; such as the viewing angle $\lambda$, that are still out of the jamming system control. Compensation techniques for the effects of such factors have to be developed.

\section{REFERENCES}

[1] S. A. VAKIN and L. N. Shustov, "Principles of Jamming and Electronic Reconnaissance", Soviet Radio, Translated by the American Intelligence in 1970.

[2] Leroy Van Brunt, "Applied ECM, Volume I", EW Engineering, Inc., 1980.

[3] G. Biernson, “Optimal Radar Tracking Systems", John Weiley \& Sons, Inc., 1990.

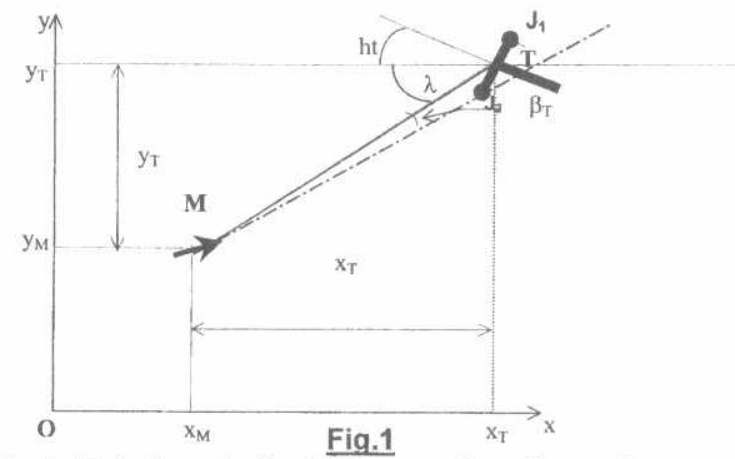

Basic Relations in the Guidance Coordinate System 


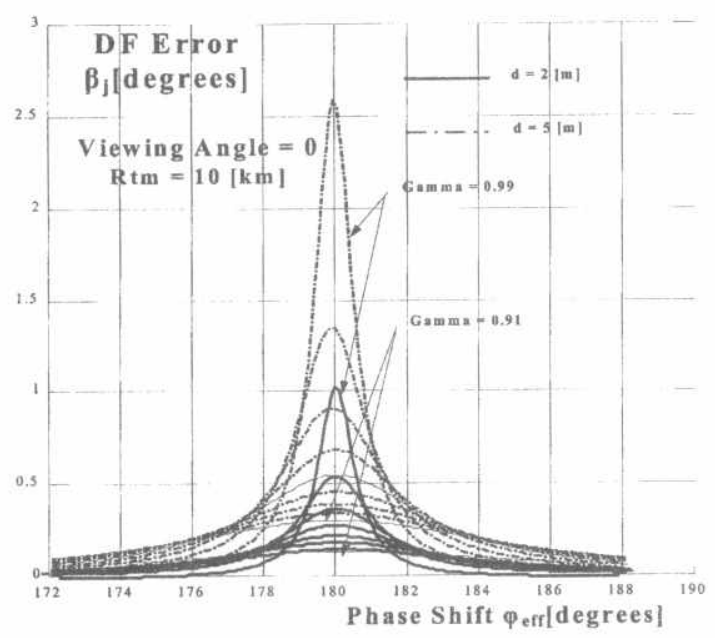

Fig. 2 Variation $\beta_{J}$ with $\varphi_{\text {Eff }}$ and $\gamma$ at two different values of $d$

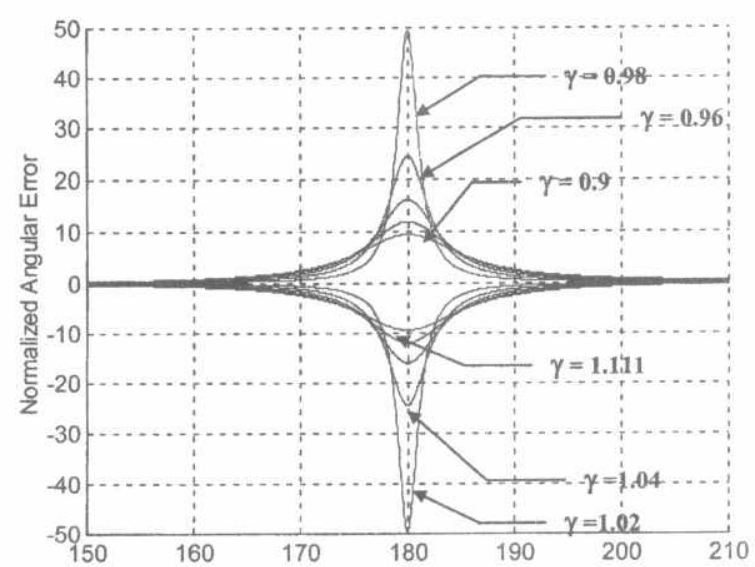

Fig. 3 Normalized Induced Jamming Error vs. Effective Phase Difference 


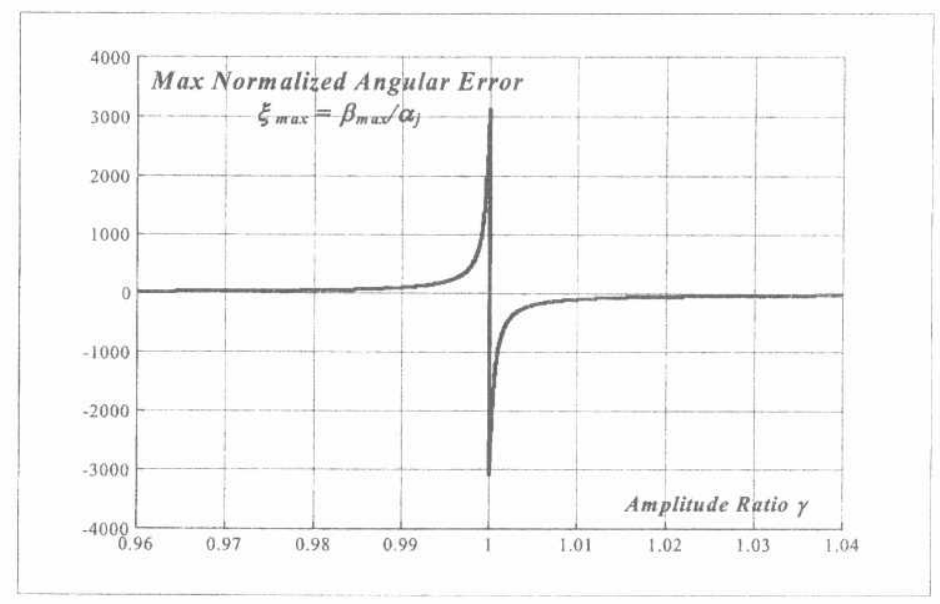

Fig. 4 Maximum value of the normalized induced error vs. the amplitude ratio $\gamma$
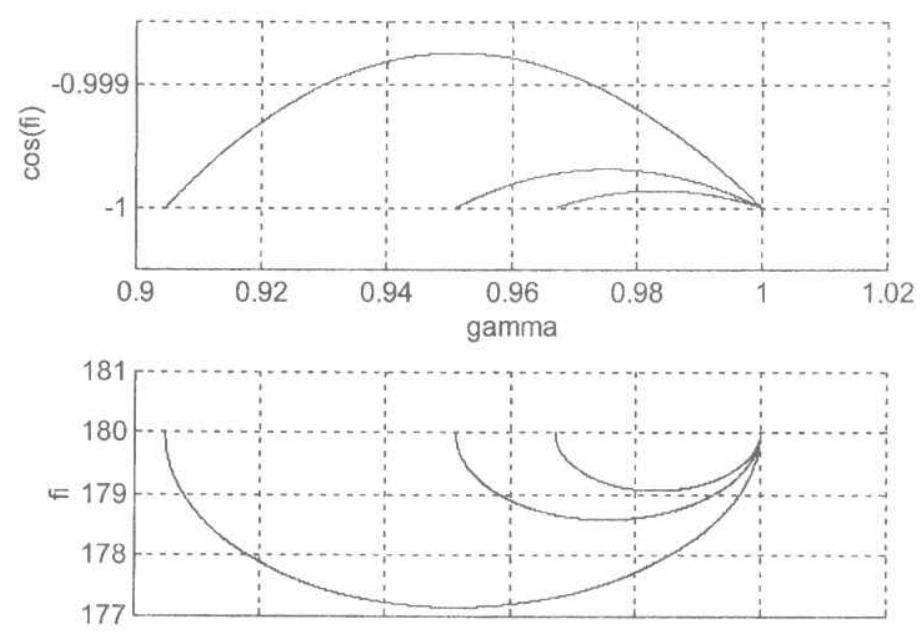

Fig. 5 Getting the permissible variation range of $\varphi_{\text {eff }}$ and $\gamma$ to guarantee a certain value for $\xi$ 

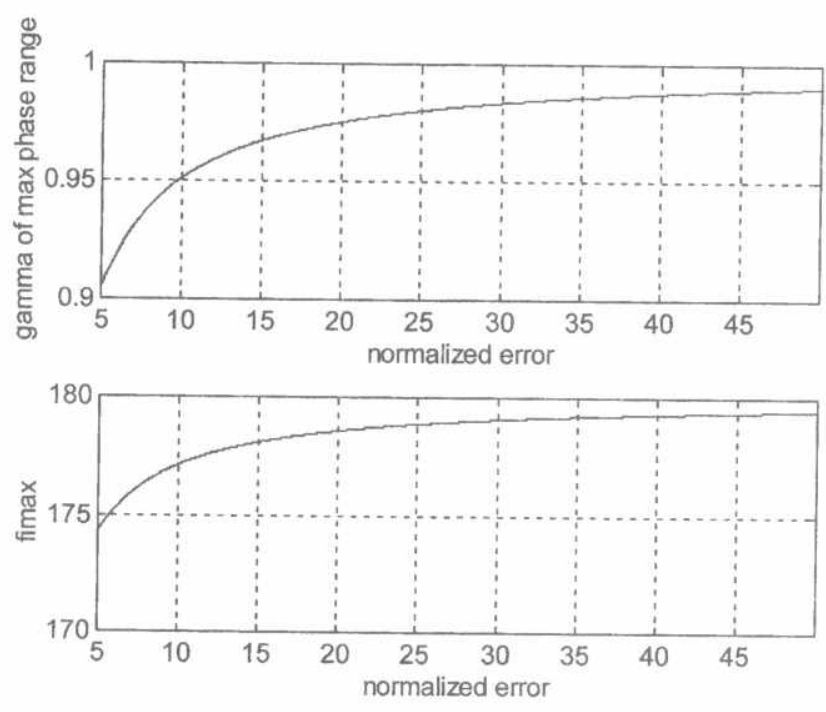

Fig. 6

Values of $\gamma$ for maximum $\varphi_{\text {eff }}$ variation at different values for $\xi$

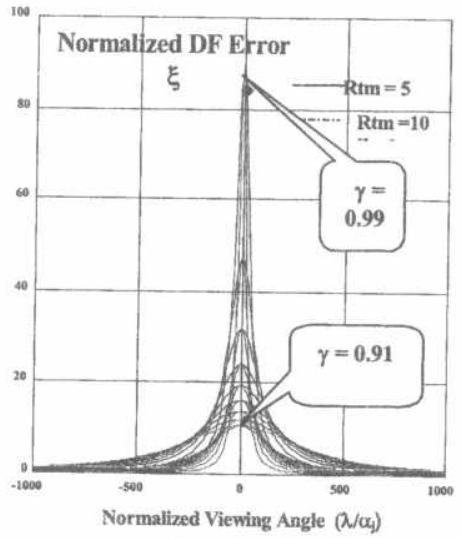

Fig. $7 \mathrm{a}$

Variation of $\xi$ with normalized viewing angle

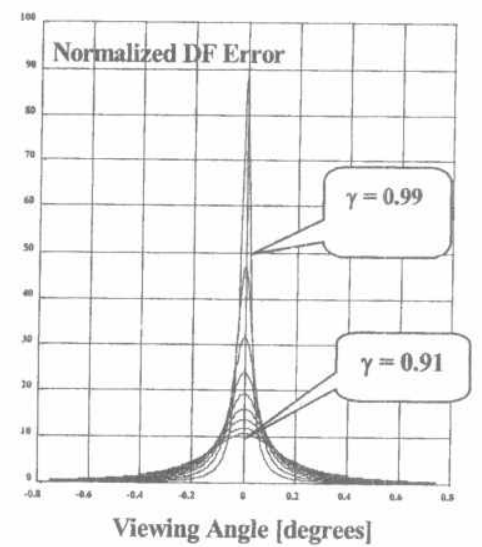

Fig. $7 \mathrm{~b}$

Variation of $\xi$ with $\lambda$

(Range Invariant) 

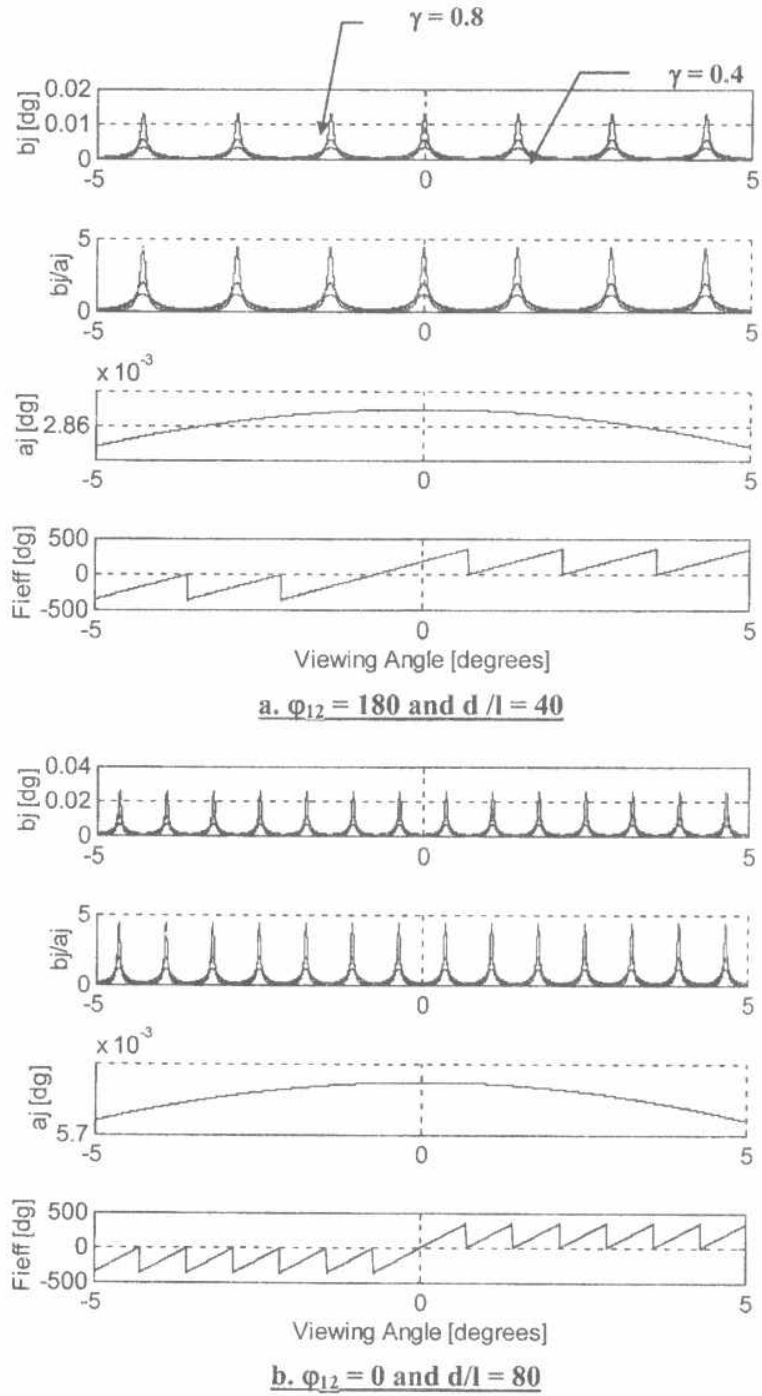

Fig. 8 Variation of the angular deception results with the viewing angle $\lambda$ 